\title{
Characterizing Geoexchange Potential Across Canada Utilizing a Multi- Layered Soil Numerical Model with a Helical Pile
}

\author{
Sarah R Nicholson ${ }^{1}$, Leya R Kober ${ }^{1}$, Seth B Dworkin ${ }^{1 *}$ \\ ${ }^{1}$ Mechanical and Industrial Engineering, Ryerson University, Toronto, Canada \\ *seth.dworkin@ryerson.ca
}

\begin{abstract}
Ground Source Heat Pumps (GSHP) have the potential to provide renewable thermal energy by utilizing the natural heat source and sink of ground conditions - significantly reducing energy demands and greenhouse gas emissions in the building sector. This energy system is particularly valuable for cold/seasonal climates such as Canada, as its operation is maintained by near constant soil conditions, and is less influenced by seasonal variations than other renewable systems. This growing field is limited by the economic and space requirements of conventional ground heat exchanger (GHE) equipment - therefore the GHE component has been the focus of much innovation. The dual use of existing foundational equipment as a GHE to provide thermal - as well as structural function for buildings is one such modification which could save material and installation costs, and increase the range of applications for a GSHP system.

These foundational piles are shallower than conventional vertical GHEs, and so they are particularly vulnerable to soil conditions. However, detailed soil characterization has been mostly utilized on a per-project basis, and there is a lack of a general and accessible categorization of soil properties in the context of thermal capacity for GSHP systems. Furthermore, numerical modelling and GHE design tends to utilize a constant, generalized soil thermal conductivity - which can lead to over or under sizing of equipment, and affect the efficiency and performance of the GSHP. Therefore, this study focused on the development of a methodology to characterize the soil thermal conductivity by depth across eleven national zones. These zones were created to synthesize trends in soil type, and climate conditions - both of which are key variables in the performance of the GSHP system.

Utilizing a numerical model of a shallow helical steel pile GHE, simulations were conducted for each region for dry, wet, and seasonal soil conditions. The steady state capacity of each pile was then calculated to present trends for researchers and industry. Ultimately, this research creates a necessary synthesis of geotechnical and climate information within the mechanical engineering field - wherein the modelling more closely represents the actual variation of conditions in which GSHP systems operate. This research provides a novel methodology for simulation of heat exchange in multi-layered soil, as well as indications for locations with the highest thermal energy potential for this renewable system - providing a foundation for further implementation of this renewable energy system across all regions of Canada.
\end{abstract}

